\title{
Recent advances in stereoselective organic reactions in aqueous media
}

\author{
$\underline{\text { Majid M. Heravi }}{ }^{1}$, Vahideh Zadsirjan, Khadijeh Kamjou \\ ${ }^{1}$ Department of Chemistry, Faculty of Physics \& Chemistry Alzahra University, Vanak, Tehran, Iran.
}

\begin{abstract}
In recent years, utilizing water as a clean, safe, readily available and almost free of charge solvent for the syntheses of a broad spectrum of organic compounds has been comprehensively realized and proven. At first glance, it seems that water is proposed as a green replacement for the harmful and evaporable organic solvents; however, in some cases, it presents exclusive reactivity and selectivity, which could not be achieved by using conventional organic solvents. Thus, using it for induction of stereoselectivity is absoloutly vital. At this juncture, performing stereoselective organic syntheses in either water or aqueous media has been the center of attention due to remarkable and unique results and a variety of these types of reactions have been effectively and successfully conducted. In this review, we try to highlight the recent stereoselective organic reactions, performed and developed, successfully in aqueous media. Dedicated to Professor Minoo Dabiri, an Iranian scientist woman, for her continuous efforts and achievements, in the field of organic chemistry.
\end{abstract}

\section{Keyword}

Aqueous media, Asymmetric synthesis, Green chemistry, Stereoselectivity, Multicomponent reaction 